\title{
Beam Quality of Multicore Fibre Lasers
}

\author{
M. Kochanowicz*, D. Dorosz, J. Żmojda And J. Dorosz \\ Białystok University of Technology, Wiejska 45D, 15-351 Białystok, Poland
}

\begin{abstract}
In the paper the beam quality of the phase-locked multicore fibre lasers was investigated. The beam quality factor (BQF) of the coherently combined beam of the multicore fibre lasers should be determinate as the laser optical output power in a central peak far-field bucket divided by the total optical output power radiating from the effective near-field. Classical $M^{2}$ factor is not proper for evaluating the beam quality of phase-locked multicore fibre lasers because it degrades with the increasing number of cores. The beam quality factor of the manufactured multicore fibres equals: 7-core hexagonal structure fibre $(\mathrm{BQF}=0.71, V=2.4), 5$-core $(\mathrm{BQF}=0.70, V=2.4)$, 30 -core circular structure of core optical fibre laser possesses the lowest beam quality factor $(\mathrm{BQF}=0.48$, $V=2.4)$. However, the angular divergence of the central peak is reduced in proportion to the number of cores generating mutually coherent radiation. Numerical simulation shows that standard deviation of the phase error below $15^{\circ}$ has an inconsiderable impact on the laser beam quality. The luminescence spectra of the fabricated constructions: 5-core, 7 -core and 30-core double clad multicore optical fibres doped with $\mathrm{Nd}^{3+}$ were measured.
\end{abstract}

PACS numbers: 42.81.-i, 42.79.Ag, 33.20.Kf

\section{Introduction}

The development of rare earth (RE) doped optical fibres enabled to produce fibre lasers with a very good quality of the radiation beam. The essential role is played here by double clad active optical fibre, different from the structure of the conventional one. In the classical double clad active fibre it can be collected relatively small amount of energy mainly because of thin core (single mode operation) and clustering phenomena. In order to increase RE concentration LMA fibres were developed [1-8]. The other way to increase active dopant in the optical fibres can be realised in multicore optical fibre. In double-clad multicore optical fibres the accumulated amount of active dopant ions is significantly greater than in classical single-core optical fibre. This enables to increase the ability to accumulate energy by the active medium, thus facilitating the $N$-fold reduction of the fibre length (where $N$ is the number of cores), necessary to absorb the pumping radiation. The basic problem of such a structure is, however, a low quality of the laser beam. The radiation intensity and the beam divergence in such a case is changing proportionally to the number of emitters. If radiation generated in particular cores is mutually coherent (phased), then the far-field diffraction pattern of the laser beam consists of a central high-intensity and low-divergence peak and symmetrical side lobes with considerably lower radiation intensity [9-13]. The angular divergence of the central peak is reduced in proportion to the number of emitters (elements of the matrix) generating mutually coherent radiation.

In the presented paper the beam quality of the phase-locked multicore fibre lasers was investigated. The influence of material and geometric parameters of the active multicore optical fibre and phase deviation on the quality

\footnotetext{
* corresponding author; e-mail: m.kochanowicz@pb.edu.pl
}

factor of the laser beam in the far-field diffraction region has been analysed.

\section{Far-field diffraction pattern of an active multicore optical fibre}

In the analysed optical fibres single-mode cores are placed in a common cladding. The core diameter amounts to: $2 r=10 \mu \mathrm{m}$, the normalized frequency $V=1.7-2.4$, and the distance between the cores $d=$ 18-25 $\mu \mathrm{m}$. Spatial distribution of the emitted laser radiation for each core has been described with the Gaussian function

$$
E_{m}(x, y, z=0)=A_{m} \exp \left(\frac{r^{2}}{w_{0}^{2}}+\mathrm{i} \varphi_{m}\right),
$$

where $A_{m}$ - maximum field amplitude in the $m$-th core, $r^{2}=\left(x-m x_{d}\right)^{2}+\left(y-n y_{d}\right)^{2}-$ coordinates of core in the multicore fiber, $w_{0}^{2}$ - radius of the mode field, $\varphi_{m}$ - phase of radiation generated in the $m$-th core.

Distribution of the field amplitude in the multicore optical fibre can be described by the following formula:

$$
E(x, y, z)=\sum_{m} A_{m}(z) E_{m}(x, y) \mathrm{e}^{\mathrm{i} \beta_{m} z} .
$$

A complete description of the laser beam generated by the $n$-th core in the plane can be found in the following equation:

$$
\begin{aligned}
& E_{n}(x, y, z)=\frac{w_{0}}{w(z)} \exp \left(-\frac{\left[\left(x-x_{n}\right)^{2}+\left(y-y_{n}\right)^{2}\right]}{w^{2}(z)}\right) x \\
& \quad \times \exp \left(-\mathrm{i}\left(k\left(\frac{\left[\left(x-x_{n}\right)^{2}+\left(y-y_{n}\right)^{2}\right]}{2 R(z)}+z\right)\right.\right. \\
& \left.\left.-\Psi+\Psi_{n 0}\right)\right),
\end{aligned}
$$

where $\lambda$ - wavelength of the laser radiation, $\left(x_{n}, y_{n}\right)$ coordinates of the central core, $\Psi_{n 0}$ - initial phase of radiation of the $n$-th core, 


$$
\begin{aligned}
& k=\frac{2 \pi}{\lambda}, \quad Z_{0}=\frac{\pi w_{0}^{2}}{\lambda}, \quad w(z)=w_{0} \sqrt{1+\left(z / z_{0}\right)^{2}} \\
& R(z)=Z_{0}\left(\frac{z}{Z_{0}}+\frac{Z_{0}}{z}\right), \quad \Psi=\arctan \left(z / z_{0}\right) .
\end{aligned}
$$

Assuming that coupling occurs only between adjacent cores, the field distribution, $E_{m}$, in each core is the sum of the power conducted in the core and the power resulting from coupling with adjacent cores described by the formula

$$
\frac{\mathrm{d} E_{m}}{\mathrm{~d} z}=-\mathrm{i} \beta_{m} A_{m}(z) \mathrm{e}^{-\mathrm{i} \beta_{m} z}+\sum_{n} C_{m, n} A_{n}(z) \mathrm{e}^{-\mathrm{i} \beta_{n} z},
$$

where $A_{m}(z)$ - the field amplitude in the $m$-th core, $C$ - coupling coefficient, $\beta$ - propagation constant. The far-field diffraction pattern (in the Fraunhofer diffraction region) is described by the following formula:

$$
\begin{gathered}
U\left(x, y, z_{d}\right)=\frac{\mathrm{e}^{-\mathrm{i} k z_{d}}}{\mathrm{i} \lambda z_{d}} \iint U\left(x_{1}, y_{1}, z_{1}\right) \\
\times \mathrm{e}^{\frac{-\mathrm{i} k}{2 z_{d}}\left[\left(x-x_{d}\right)^{2}+\left(y-y_{d}\right)^{2}\right]} \mathrm{d} x_{1} \mathrm{~d} y_{1},
\end{gathered}
$$

where $k=\frac{2 \pi}{\lambda}, \lambda$ - wavelength, $x_{1}, y_{1}$ - location coordinates of the cores, $z_{d}$ - observation distance.

The far-field intensity pattern is proportional to the square of the field amplitude averaged in time $|U(x, y, z)|^{2}$. A direct integration of the above equation may be complicated. In order to calculate the far-field pattern a method based on the two-dimensional fast Fourier transform (FFT) has been used [14].

\section{Beam quality factor of the laser beam emitted by a mutually coherent emitter matrix}

The value of the classical parameter, $M^{2}$, describing the quality of the laser beam for a matrix of mutually coherent lasers is proportional to the number of emitters [15]. It follows that the $M^{2}$ parameter does not

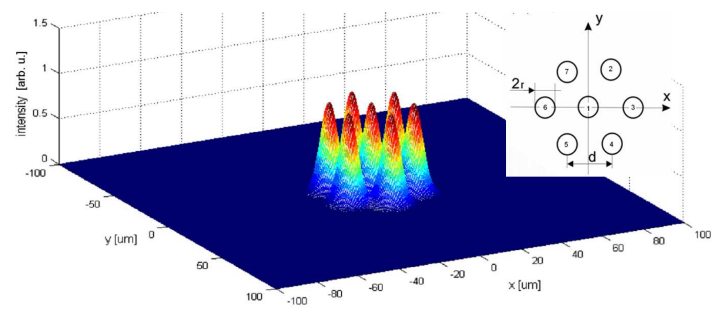

Fig. 1. Near-field pattern and arrangement of cores (inset) of 7-core optical fibre, $V=2.4, d=18 \mu \mathrm{m}$.

describe explicitly the laser beam quality in the far-field region generated by the matrix of lasers emitting mutually coherent radiation. In the paper [15] a new parameter (beam propagation factor) has been suggested, which describes in a quantitative way the quality of the laser beam emitted by the matrix of phase-locked emitters. It

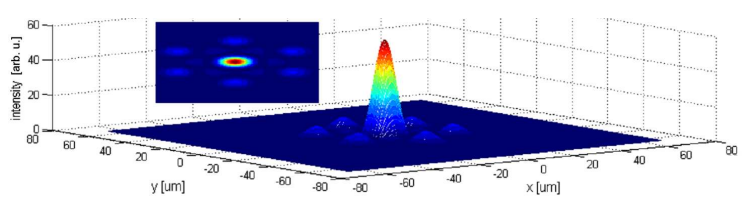

Fig. 2. Far-field pattern of 7-core phase-locked optical fibre, $V=2.4, d=18 \mu \mathrm{m}$.

is defined as a ratio of the refracting power in the central peak to the total refracting power of the beam in the near field. The suggested parameter is dependent only on the filling factor of the matrix of emitters $(t)$, not on their number. In order to describe compactness of the emitter matrix the parameter $t=(d-2 r) / 2 r$ has been introduced. The matrix of emitters arranged close to one another is characterised by a lower value of the $t$ parameter. The influence of material and geometric parameters of the active multicore optical fibre on the quality factor of the laser beam has been analysed.

The divergence between phases of radiation generated in the cores of the multicore optical fibre operating in the fibre laser system has been described by means of the standard deviation

$$
\mathrm{SD}=\sqrt{\frac{1}{N} \sum_{i=1}^{N}\left(\Theta_{i}-\bar{\Theta}\right)^{2}},
$$

where $\Theta_{i}$ - radiation phase in the $i$-th core, $\bar{\Theta}=$ $(1 / N) \sum_{i=1}^{N} \Theta_{i}-$ mean value of phases of radiation generated in all cores, $N$ - number of emitters.

The impact of phase deviations occurring as a result of various external factors on the laser beam quality in the far-field region has also been analysed.

\subsection{Active 7-core optical fibre}

The near-field and arrangement of cores in the analyzed 7-core optical fiber are presented in Fig. 1. Figures 2, 3 and 4 show results of the far-field pattern

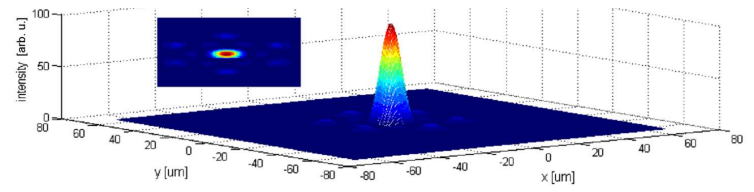

Fig. 3. Far-field pattern of 7-core phase-locked optical fibre, $V=2, d=18 \mu \mathrm{m}$.

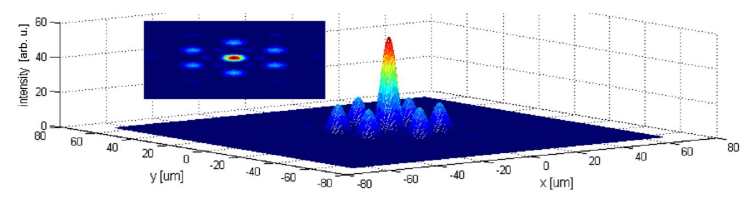

Fig. 4. Far-field pattern of 7-core phase-locked optical fibre, $V=2.4, d=25 \mu \mathrm{m}$. 


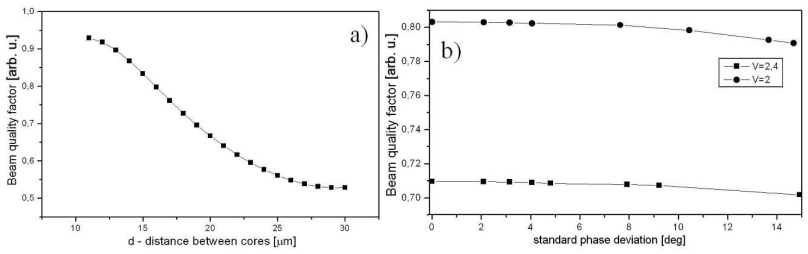

Fig. 5. Beam quality factor vs. distance between cores (a) and standard phase deviation (b).

simulation, assuming that radiation phases generated in particular cores are equal.

The central peak intensity is much higher than in case of emitters working independently. Along with the increase in the distance between the cores the amount of energy found in side lobes in the far-field pattern rises, which results in a lower quality factor of the laser beam.

The improved beam quality can be also obtained by reducing the value of normalised frequency. Then, the intensity of the central peak is higher and the amount of energy contained in the side lobes is lower. Figure $5 \mathrm{~b}$ shows the impact of incomplete phase equalization of the radiation generated in particular cores on the beam quality factor in the far field. For the value of the standard phase deviation below $15^{\circ}$, the value of the quality factor in the Fraunhofer diffraction region does not fall more than by $5 \%$.

\subsection{Active 5-core optical fibre}

Figures 6-9 below show far-field patterns of a 5-core optical fibre generating mutually coherent radiation. In comparison to the 7 -core fibre the central peak intensity is lower, and the laser spot diameter is bigger. While preserving the same distance between the cores a lower quality beam has been obtained. The amount of energy contained in side lobes is larger in case of the analysed 5 -core optical fibre. It should be also noted that the arrangement of side lobes is analogical to the arrangement of cores in the optical fibre.

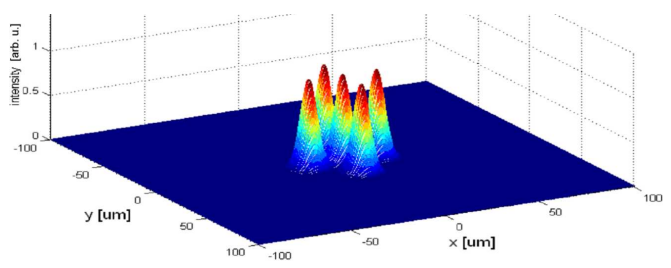

Fig. 6. Near-field pattern of 5-core optical fibre, $V=2.4, d=18 \mu \mathrm{m}$.

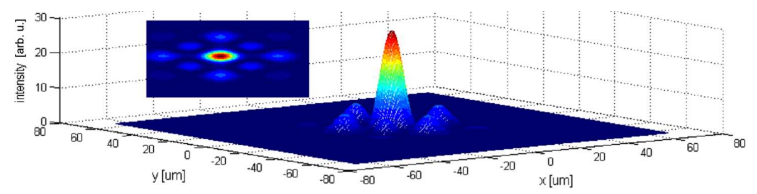

Fig. 7. Far-field pattern of 5-core phase-locked optical fibre, $V=2.4, d=18 \mu \mathrm{m}$.

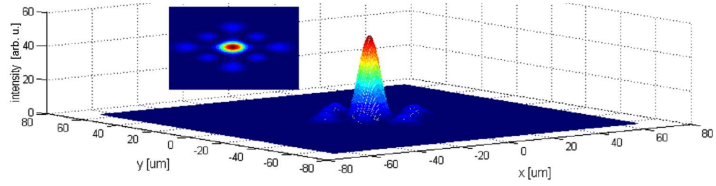

Fig. 8. Far-field pattern of 5-core phase-locked optical fibre, $V=2, d=18 \mu \mathrm{m}$.

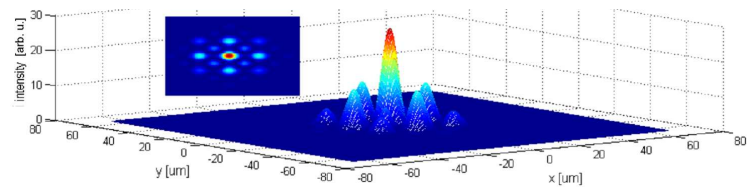

Fig. 9. Far-field pattern of 5-core phase-locked optical fibre, $V=2.4, d=25 \mu \mathrm{m}$.

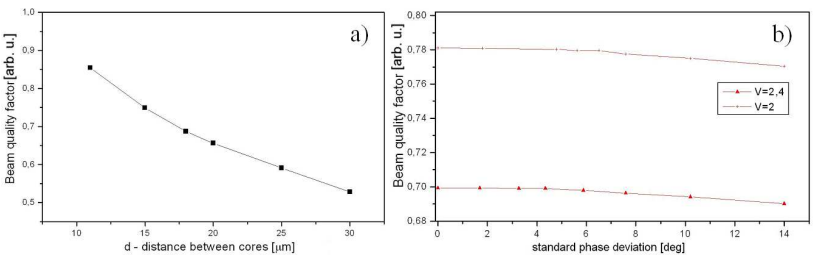

Fig. 10. Beam quality factor vs. distance between cores (a) and standard phase deviation (b).

Effect of phase deviation from ideal phase-locked multicore fibre laser on beam quality factor in the analysed above 5-core optical fibre is similar like in case 7-core optical fibre (Sect. 3.1). Relative decline of beam quality factor in the examined range is less than $1.5 \%$ (Fig. 10b).

\subsection{Active 30-core optical fibre}

The near-field of the 30-core optical fiber is shown in Fig. 11. Figures 12 and 13 show the far-field pattern simulation in the Fraunhofer diffraction region of an active 30 -core optical fibre generating mutually coherent radiation.

In comparison to 5-core and 7-core optical fibres the central peak intensity is considerably higher, while its divergence is several times lower. The side lobes are characterised by circular symmetry. However, the calculated quality factor of the laser beam is worse than in case of optical fibres analysed in points 2 and 3 . For $V=2.4$ the quality factor of the laser beam amounts to 0.48 . It means that more than half of the energy present in the far field is dispersed in the side lobes.

It should be also noted that phase errors described by means of the standard deviation for the value lower than $14^{\circ}$ practically do not have any impact on the far-field pattern of the 30-core optical fibre (Fig. 14). The relative decrease in the quality factor of the laser beam in the examined range is lower than $0.2 \%$. Taking geometric and 


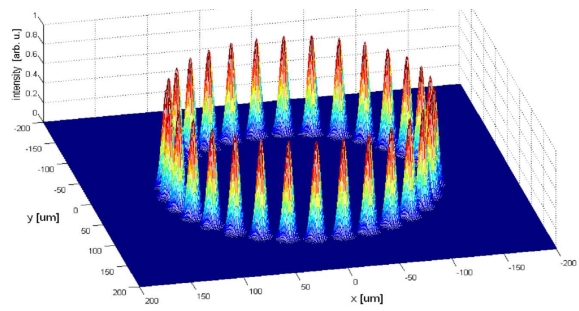

Fig. 11. Near-field pattern of 30-core optical fibre, $V=2.4, d=20 \mu \mathrm{m}$.

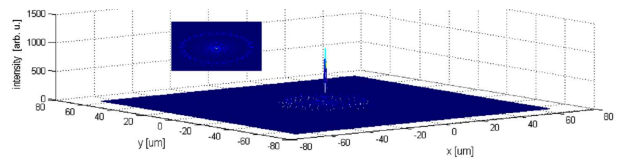

Fig. 12. Far-field pattern of 30-core phase-locked optical fibre, $V=2.4, d=20 \mu \mathrm{m}$.

material parameters of the active multicore optical fibre into consideration, the best quality of the laser beam is obtained when the value of normalised frequency and the distance between the cores are possibly low. It should be also mentioned that the laser spot is inversely proportional to the number of cores.

\section{Experiment}

The composition of the aluminosilicate glasses used for cores and internal cladding was the following: $57 \mathrm{SiO}_{2}-$ $(8-x) \mathrm{PbO}-6 \mathrm{Al}_{2} \mathrm{O}_{3}-6 \mathrm{~B}_{2} \mathrm{O}_{3}-3 \mathrm{BaO}-20\left(\mathrm{Li}_{2} \mathrm{O}+\mathrm{Na}_{2} \mathrm{O}+\right.$ $\left.\mathrm{K}_{2} \mathrm{O}\right)-x \mathrm{Nd}_{2} \mathrm{O}_{3}(x=0.15 \mathrm{~mol} . \%)$. Glasses were synthesized by conventional melting and quenching method in argon flow. Pure oxide materials $(99.99 \%)$ were used to prepare glass batches in the investigated system doped with $\mathrm{Nd}_{2} \mathrm{O}_{3}(99.99 \%)$. The glasses were melted in covered gold-platinum crucibles, in an electric furnace, at temperature $1450{ }^{\circ} \mathrm{C}$. The melted mass of glass was poured into a brass mould preheated and annealed at temperature range $400-450{ }^{\circ} \mathrm{C}$. Transparent and homogeneous glass rods without crystallization was fabricated and applied as cores in multicore double clad optical fibres. Thermal properties of the fabricated glasses were determined by differential scanning calorimeter (Setaram Labsys). Refractive index was measured on a Metricon 2010/M Prism Couple. The thermal expansion coefficient was measured by a standard dilatometer. Modified rod-in-tube drawing technique was used to fabricate dou-

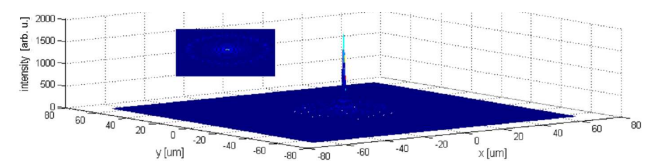

Fig. 13. Far-field pattern of 30-core phase-locked optical fibre, $V=2, d=20 \mu \mathrm{m}$.

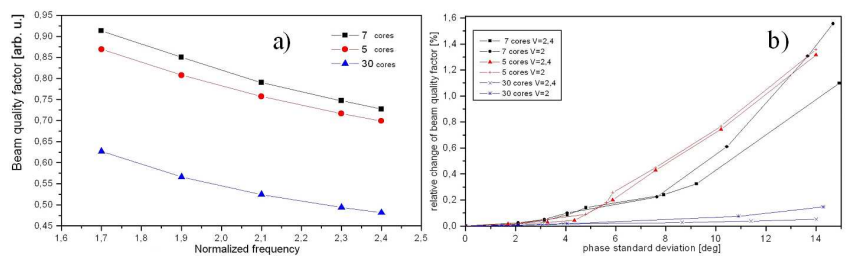

Fig. 14. Comparison of influence of normalized frequency (a) and standard deviation (b) on beam quality factor for analysed constructions of optical fibres.

ble clad, multicore optical fibres doped with $\mathrm{Nd}^{3+}$. Luminescence measurement system consisted of pumping diode HLU30FAC400-808P $\left(\lambda=808 \mathrm{~nm}, P_{\max }=30 \mathrm{~W}\right)$ with optical fibre output $(400 \mathrm{~nm}, \mathrm{NA}=0.22)$, the laser beam forming system and spectrometer (Acton Spectra Pro 2300i).

\section{Results}

Table I shows basic optical and thermal properties of the fabricated glasses for cores and cladding optical fibres.

TABLE I

Thermal and optical properties of aluminosilicate glasses.

\begin{tabular}{l|c}
\hline \hline \multicolumn{1}{c|}{ Property } & Range of values \\
\hline density $\left[\mathrm{g} / \mathrm{cm}^{3}\right]$ & $3.8-4.2$ \\
microhardness $[\mathrm{GPa}]$ & $8.5-9$ \\
mole volume $\left[\mathrm{cm}^{3} / \mathrm{mol}\right]$ & $24.6-25.8$ \\
refractive index & $1.63-1.68$ \\
transmission $[\mu \mathrm{m}]$ & $0.4-4.5$ \\
thermal expansion coefficient & $82-92$ \\
$\alpha 100-400{ }^{\circ} \mathrm{C}\left[10^{-7} 1 / \mathrm{K}\right]$ & $438-480$ \\
glass transition temperature $T_{\mathrm{g}}\left[{ }^{\circ} \mathrm{C}\right](\mathrm{DL})$ & $500-535$ \\
softening point $T_{\mathrm{s}}\left[{ }^{\circ} \mathrm{C}\right]$ & $445-458$ \\
glass transition temperature $T_{\mathrm{g}}\left[{ }^{\circ} \mathrm{C}\right](\mathrm{DSC})$ &
\end{tabular}

Below are shown results of investigation on double clad, multicore optical fibres doped with neodymium fabrication technology (Tables II-IV, Figs. 15-20).
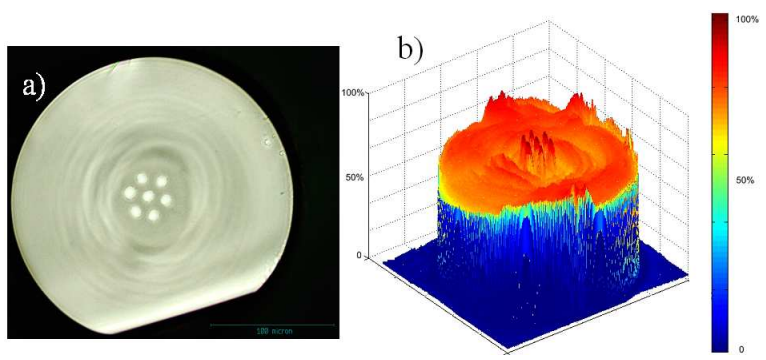

Fig. 15. Cross-section (a) of luminance distribution, (b) of the fabricated 7 -core fibre doped with $\mathrm{Nd}^{3+}$. 


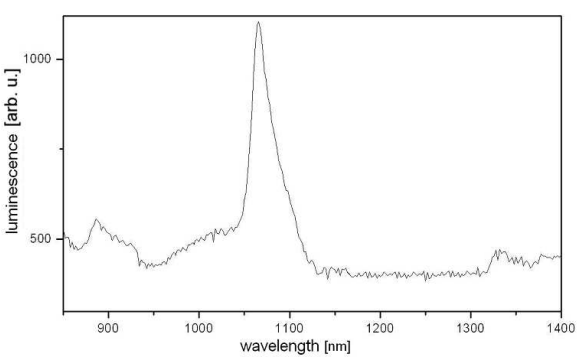

Fig. 16. Luminescence spectra of the 7-core optical fibre doped with $\mathrm{Nd}^{3+}\left(\lambda_{\mathrm{p}}=808 \mathrm{~nm}\right)$.
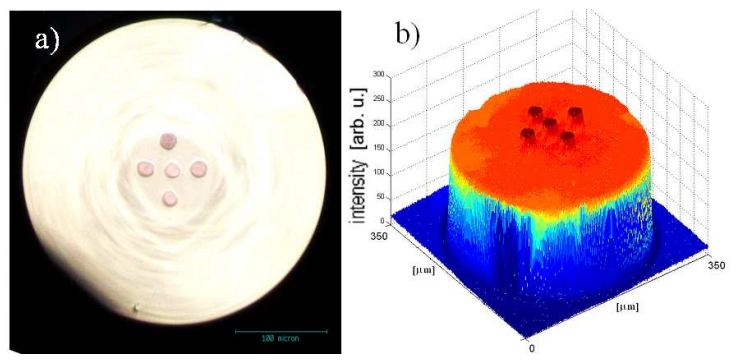

Fig. 17. Cross-section (a) of luminance distribution (b), of the fabricated 5-core fibre doped with $\mathrm{Nd}^{3+}$.

Large numerical aperture diameter of inner cladding and numerical aperture $\left(\mathrm{NA}_{\text {cladding }}=0.58\right)$ enables effective coupling pump laser diode with the obtained fibre. The luminescence spectra of the manufactured multicore fibres doped with $\mathrm{Nd}^{3+}$ under excitation through inner cladding by laser diode $\mathrm{AlGaAs}(\lambda=808 \mathrm{~nm})$ are shown in Figs. 16, 18, 20. Emission bands at $900 \mathrm{~nm}$ $\left({ }^{4} F_{3 / 2} \rightarrow{ }^{4} I_{9 / 2}\right), 1060 \mathrm{~nm}\left({ }^{4} F_{3 / 2} \rightarrow{ }^{4} I_{11 / 2}\right)$ and $1330 \mathrm{~nm}$ $\left({ }^{4} F_{3 / 2} \rightarrow{ }^{4} I_{13 / 2}\right)$ were attained. Due to four-level quantum scheme operation optical transition $\left({ }^{4} F_{3 / 2} \rightarrow{ }^{4} I_{11 / 2}\right)$ related with luminescence band at $1060 \mathrm{~nm}$ is the most efficient. However, emission at $900 \mathrm{~nm}$ and $1330 \mathrm{~nm}$, usually unparalleled in silicate glasses were also observable.

\section{Summary}

The value of the classical parameter $M^{2}$, describing the quality of the laser beam for a matrix of mutually

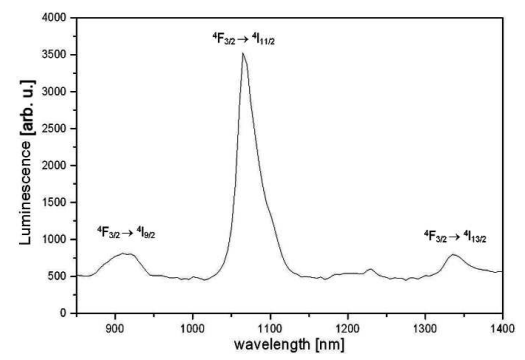

Fig. 18. Luminescence spectra of the 5-core optical fibre doped with $\mathrm{Nd}^{3+}\left(\lambda_{\mathrm{p}}=808 \mathrm{~nm}\right)$.
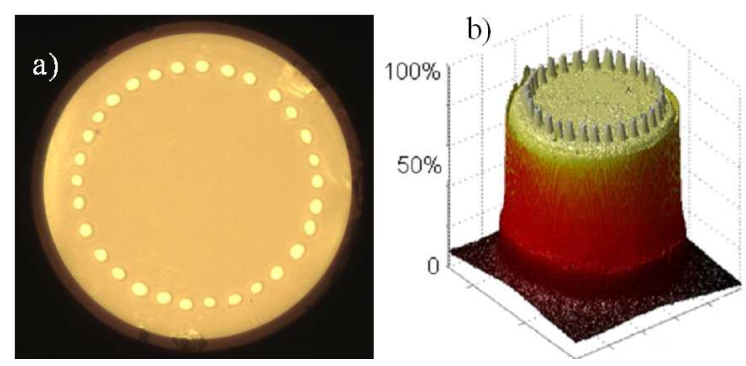

Fig. 19. Cross-section (a) of luminance distribution, (b) of the fabricated 5 -core fibre doped with $\mathrm{Nd}^{3+}$.

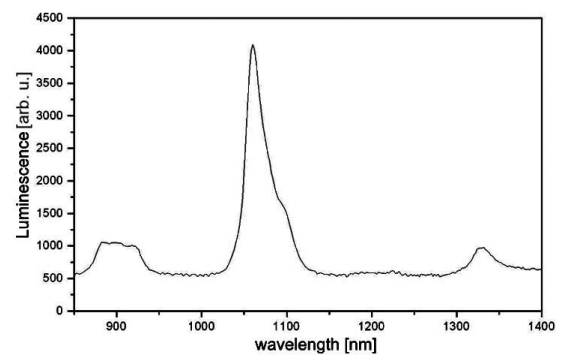

Fig. 20. Luminescence spectra of the 30-core optical fibre doped with $\mathrm{Nd}^{3+}\left(\lambda_{\mathrm{p}}=808 \mathrm{~nm}\right)$.

TABLE II

Properties of the 7-core optical fibre doped with $\mathrm{Nd}^{3+}$.

\begin{tabular}{l|c}
\hline \hline \multicolumn{1}{c|}{ Property } & Value \\
\hline outer diameter $[\mu \mathrm{m}]$ & 250 \\
diameter of cores $[\mu \mathrm{m}]$ & 10 \\
$\mathrm{NA}_{\text {cores }}$ & 0.07 \\
$\mathrm{NA}_{\text {cladding }}$ & 0.58
\end{tabular}

TABLE III

Properties of the 5-core optical fibre doped with $\mathrm{Nd}^{3+}$.

\begin{tabular}{l|c}
\hline \hline \multicolumn{1}{c|}{ Property } & Value \\
\hline outer diameter $[\mu \mathrm{m}]$ & 350 \\
diameter of cores $[\mu \mathrm{m}]$ & 13 \\
$\mathrm{NA}_{\text {cores }}$ & 0.07 \\
$\mathrm{NA}_{\text {cladding }}$ & 0.58
\end{tabular}

TABLE IV

Properties of the 30-core optical fibre doped with $\mathrm{Nd}^{3+}$.

\begin{tabular}{l|c}
\hline \hline \multicolumn{1}{c|}{ Property } & Value \\
\hline outer diameter $[\mu \mathrm{m}]$ & 350 \\
diameter of cores $[\mu \mathrm{m}]$ & 13 \\
$\mathrm{NA}_{\text {cores }}$ & 0.07 \\
$\mathrm{NA}_{\text {cladding }}$ & 0.58
\end{tabular}


coherent lasers is proportional to the number of emitters. It follows that the $M^{2}$ parameter does not describe explicitly the laser beam quality in the far-field region generated by the matrix of lasers emitting mutually coherent radiation. A new parameter (beam quality factor) has been suggested, which describes, in a quantitative way, the quality of the laser beam emitted by the matrix of phase-locked emitters. It is defined as a ratio of the refracting power in the central peak to the total refracting power of the beam in the near field. Performed numerical simulations unequivocally show that the best quality of the laser beam is obtained when the value of normalised frequency and the distance between the cores are possibly low. Arrangement of cores in multicore optical fibre also influences on beam quality. The highest value of beam quality $(\mathrm{BQF}=0.71)$ was attained in 7-core hexagonal-arrangement optical fibre $(V=2.4$, $d=18 \mu \mathrm{m})$, whereas construction with a circular fibre cores distribution is characterised by the lowest value of the quality of the laser beam $(\mathrm{BQF}=0.48)$. In this case more than half energy in the far-field bucket, is dispersed in the side lobes.

In comparison to construction 5- and 7-core optical fibre intensity of the central peak (supermode) is significantly higher, while divergence several times smaller. Diameter of spot is inversely proportional to the number of active cores. Performed calculations of the impact of phase deviation from ideal phase-locked multicore fibre laser indicate that standard deviation of phase below $14^{\circ}$ has practically no impact on the far-field diffraction patterns of the analysed active multicore optical fibres. Relative decline of beam quality factor in the considered range is less than $2 \%$ for 5 - and 7-core optical fibres and less than $0.2 \%$ in the case of 30 -core optical fibre. Designed and manufactured multicore fibres doped with $\mathrm{Nd}^{3+}$ are characterized by strong luminescence at $1060 \mathrm{~nm}$. There is a possibility of their application as an active medium for constructing short length, high power fibre lasers utilizing diffractive coupling of radiation from the cores into a supermode.

\section{Acknowledgments}

This work was supported by the Ministry of Science and Higher Education of Poland - grant No. R08 022 02 and Białystok University of Technology - grant No. $\mathrm{W} / \mathrm{WE} / 4 / 2010$.

\section{References}

[1] A. Zając, J. Świderski, P. Konieczny, S. Gągała, High Power Fibre Lasers - Analysis and Construction Requirements, MUT, Warsaw 2008.

[2] A. Desfarges-Berthelemot, V. Kermene, D. Sabourdy, J. Boullet, P. Roy, J. Lhermite, A. Barthélémy, C.R. Phys. 7, 244 (2006).

[3] Y. Kono, M. Takeoka, K. Uto, A. Uchida, F. Kannari, IEEE J. Quant. Electron. 36, 607 (2000).

[4] Y. Li, L. Qiana, D. Lua, D. Fana, S. Wenb, Opt. Laser Technol. 39, 957 (2007).

[5] S. Serati., H.A. Masterson Linnenberger, Proc. 2004 IEEE 3, 6 (2004).

[6] M. Wrage, P. Glas, M. Leitner, D.V. Vysotsky, A.P. Napartovich, Opt. Commun. 191, 149 (2001).

[7] K. Barczak, T. Pustelny, D. Dorosz, J. Dorosz, Acta Phys. Pol. A 116, 247 (2009).

[8] K. Barczak, T. Pustelny, Z. Zycki, T. Blazejczak, Acta Phys. Pol. A 116, 250 (2009).

[9] T. Pustelny, M. Grabka, Acta Phys. Pol. A 116, 385 (2009).

[10] T. Pustelny, M. Grabka, Acta Phys. Pol. A 114, A-113 (2009).

[11] D. Dorosz, M. Kochanowicz, Proc. SPIE $\mathbf{7 1 2 0}$ $71200 \mathrm{H}(2008)$.

[12] M. Kochanowicz, D. Dorosz, J. Żmojda, Proc SPIE 7502, 25 (2009).

[13] M. Kochanowicz, D. Dorosz, A. Zając, Electronics 10, 151 (2009).

[14] J. Petykiewicz, Wave Optics, PWN, Warsaw 1986.

[15] P. Zhou, Z. Liu, X. Xu, Z. Chen, X. Wang, Opt. Laser Technol. 41, 268 (2009). 